\title{
Streamlined design of a self-inactivating feline immunodeficiency virus vector for transducing ex vivo dendritic cells and $T$ lymphocytes
}

\author{
Mauro Pistello*, Laura Vannucci, Alessia Ravani, Francesca Bonci, \\ Flavia Chiuppesi, Barbara Del Santo, Giulia Freer and Mauro Bendinelli
}

Address: Retrovirus Center and Virology Section, Department of Experimental Pathology, University of Pisa, Pisa, Italy

Email: Mauro Pistello* - pistello@biomed.unipi.it; Laura Vannucci - lauravannucci@biomed.unipi.it;

Alessia Ravani - alessiaravani@biomed.unipi.it; Francesca Bonci - f.bonci@kedrion.com; Flavia Chiuppesi - flo83@email.it; Barbara Del

Santo - barbaradelsanto@biomed.unipi.it; Giulia Freer - freer@biomed.unipi.it; Mauro Bendinelli - bendinelli@biomed.unipi.it

* Corresponding author

Published: 19 September 2007

Genetic Vaccines and Therapy 2007, 5:8 doi:10.1186/1479-0556-5-8

This article is available from: http://www.gvt-journal.com/content/5/l/8

(C) 2007 Pistello et al; licensee BioMed Central Ltd.

This is an Open Access article distributed under the terms of the Creative Commons Attribution License (http://creativecommons.org/licenses/by/2.0), which permits unrestricted use, distribution, and reproduction in any medium, provided the original work is properly cited.

\begin{abstract}
Background: Safe and efficient vector systems for delivering antigens or immunomodulatory molecules to dendritic cells (DCs), T lymphocytes or both are considered effective means of eliciting adaptive immune responses and modulating their type, extent, and duration. As a possible tool toward this end, we have developed a self-inactivating vector derived from feline immunodeficiency virus (FIV) showing performance characteristics similar to human immunodeficiency virus-derived vectors but devoid of the safety concerns these vectors have raised.
\end{abstract}

Methods: The pseudotyped FIV particles were generated with a three-plasmid system consisting of: the packaging construct, providing Gag, Pol and the accessory proteins; the vector(s), basically containing FIV packaging signal $(\psi)$, Rev responsive element, R-U5 region at both ends, and the green fluorescent protein as reporter gene; and the Env plasmid, encoding the G protein of vesicular stomatitis virus (VSV-G) or the chimeric RDII4 protein. Both packaging and vector constructs were derived from P34TFI0, a replication competent molecular clone of FIV. The pseudotyped particles were produced by transient transfection in the Crandell feline fibroblast kidney (CrFK) or the human epithelial (293T) cell line.

Results: To broaden its species tropism, the final vector construct was achieved through a series of intermediate constructs bearing a longer $\psi$, the FIV central polypurin tract sequence (cPPT), or the woodchuck hepatitis postregulatory element (WPRE). These constructs were compared for efficiency and duration of transduction in $\mathrm{CrFK}$ or 293T cells and in the murine fibroblast cell line $\mathrm{NIH}-3 \mathrm{~T} 3$. Whereas $\psi$ elongation and cPPT addition did not bring any obvious benefit, insertion of WPRE downstream GFP greatly improved vector performances. To maximize the efficiency of transduction for ex-vivo murine DCs and T-lymphocytes, this construct was tested with VSV-G or RDII4 and using different transduction protocols. The results indicated that the FIV construct derived herein stably transduced both cell types, provided that appropriate vector makeup and transduction protocol were used. Further, transduced DCs underwent changes suggestive of an induced maturation.

Conclusion: In contrast to previously described FIV vectors that were poorly efficient in delivering genetic material to DCs and T lymphocytes, the vector developed herein has potential for use in experimental immunization strategies. 


\section{Background}

Upon encountering foreign invaders, dendritic cells (DCs) in the periphery of the body undergo a dynamic and coordinated reprogramming of gene expression, surface phenotype and cellular function [1]. While this maturation is ongoing, DCs migrate to lymphoid organs where they interact with T lymphocytes which, in turn, decode the DC message to start a cascade of events ultimately leading to immune responses against the invading antigens. Thus, at least theoretically, safe and effective systems for delivering antigenic and/or adjuvant proteins/ genes to DCs, T cells or both represent valuable means of eliciting and modulating type, extent, and duration of adaptive immune responses [2]. Although initial attempts to achieve this goal using conventional methods were disappointing, recent advances have opened new and more promising avenues $[3,4]$.

Viruses are considered ideal for delivering transgenes due to their inherent ability to bring genetic material into cells but need extensive engineering to overcome limitations such as the spectrum of cells they can enter and the noxious effects they may exert. For example, adenoviral vectors have been shown to be effective at transducing DCs and $\mathrm{T}$ lymphocytes [5] but, on a negative side, they have been seen to induce massive production of proinflammatory cytokines and robust vector-specific immune responses [6]. On the other hand, oncoretroviral vectors interfere minimally with normal body and cell functions but are poor at transducing nondividing and rarely dividing cells, such as DCs and resting T cells.

Consistent with the ability of lentiviral genomes to reach the nucleus of host cells even if these do not divide [7], vectors derived from the human immunodeficiency virus (HIV) have been found to transduce DCs and T cells at high efficiency [8-11]. In their current versions, HIV vectors have most of the original viral genome deleted, including some transcriptional elements in the $\mathrm{U} 3$ region of the 3' long terminal repeat (LTR) of the DNA used to produce the vector RNA. During reverse transcription, this deletion is transferred to the 5' LTR of the proviral DNA, thus generating two LTRs which are mostly inactive (selfinactivating $[\mathrm{SIN}]$ vectors). Also, these vectors are produced using multiple constructs encoding different components to minimize the risk of generating replicationcompetent viruses.

Because of safety concerns [12], vectors derived from the feline immunodeficiency virus (FIV) are considered a good alternative to the HIV vectors because FIV has never been detected in animal species other than domestic and wild cats and has similar genome organization but minimal sequence homology to HIV, thus minimizing the risk of unwanted recombinations [13].
The FIV vectors described to date have been very successful at delivering transgenes into a variety of cells of different animal species [reviewed in [12]] but have performed poorly when used to transduce DCs, T lymphocytes and non-adherent white blood cells in general [14-16]. In this report, we describe a SIN FIV vector that effectively transduces ex vivo murine DCs and T cells.

\section{Methods \\ Parental plasmid and strategy used for packaging and vector construction}

Prototype vectors and packaging construct were developed from $\mathrm{p} \Delta 00$ (Fig. 1A and $2 \mathrm{~A}$ ), a replication-competent molecular clone of the Petaluma strain of FIV (FIVPet), derived from plasmid p34TF10 [GenBank: NC 001482] and produced in our laboratory by substituting a tryptophan codon for the stop codon in the accessory gene ORF-A [17]. As reported [18], an intact ORF-A is essential for optimal FIV replication in lymphoid cells. Nucleotide (nt) positions of packaging and vector constructs are referred to the NC 001482 sequence. The sequence of primers used in polymerase chain reaction (PCR) is available by e-mail on request. All the intermediate and final constructs described below were checked for proper insertions and absence of unwanted mutations by cycle sequencing using an automated DNA sequencer (GE Healthcare, Milan, Italy). All vectors were tested using enhanced green fluorescent protein (GFP) as reporter molecule.

\section{Packaging constructs}

Four packaging constructs were developed and tested. Due to minimal activity of the FIV LTR in non feline cells [19], all constructs had the 5' and 3' LTRs of p $\Delta 00$ replaced with cytomegalovirus early promoter (CMVp) and bovine growth hormone poly A, respectively. Also, deletion of the 5'LTR was extended to nt position 507 to remove most of the RNA packaging site $(\psi)$. From this intermediate construct the packaging constructs were produced as follows:

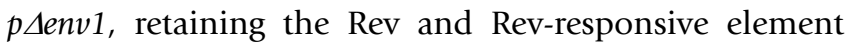
(RRE), necessary for the nuclear export of Gag-Pol mRNA, and containing an internal 1,044 nt deletion within env (nt position 7246-8289) obtained by digestion with the restriction enzymes $B c l$ I and SpeI (New England Biolabs, Celbio, Milan, Italy), filling up the protruding ends with Klenow DNA polymerase (New England Biolabs) and joining of resulting blunt-ends with T4 DNA ligase (Fermentas, M-Medical, Milan, Italy) (Fig. 1B).

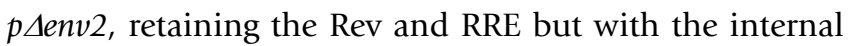
env deletion extended from end of the first exon Rev to beginning of RRE (nt position 7246-8289). This deletion was created by PCR using overlapping primers (Fig. 1C). 
A

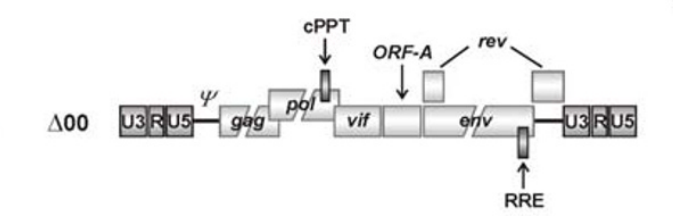

B P Denv1
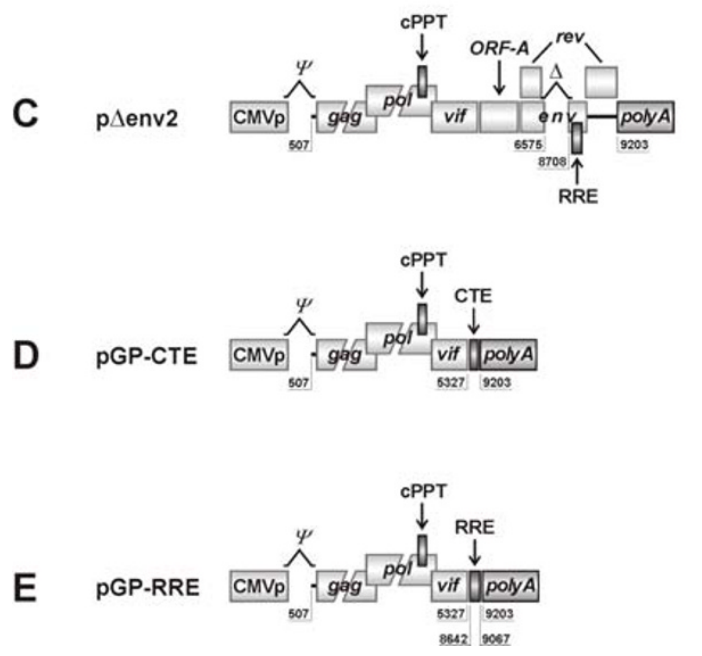

5494

5713

\section{Figure I}

Schematic representation of the FIV packaging constructs used. A) Parental clone $\mathrm{p} \Delta 00$. B) Packaging construct $\mathrm{p} \Delta$ env l; the $5^{\prime}$ and $3^{\prime}$ LTRs are replaced by $C M V P$ and bovine growth hormone (BGH) poly A, respectively, $\psi$ is partially deleted, and env is deleted by an internal I Kbp. C) Packaging construct $\mathrm{p} \Delta$ env2; derived from $\mathrm{p} \Delta$ env l by removing the entire env except for the terminal ends overlapping the first exon rev and the Rev-responsive element (RRE) necessary for nuclear export of unspliced viral RNAs; D) Packaging construct $P G P-C T E$; obtained by deleting from within vif to $B G H$ poly $A$, and replacing the Rev-RRE system with the Mason-Pfizer monkey virus constitutive transporting element (CTE); E) Packaging construct pGP-RRE; derived from pGPCTE by replacing the CTE with the RRE retrieved by amplification from the parental clone $\mathrm{p} \Delta 00$. For this construct, Rev was provided in trans.

pGP-CTE, containing a truncated vif and deleted of ORF$\mathrm{A}, e n v, r e v$ and RRE. This genome portion was removed by digestion with the restriction enzymes BspMII (nt position 5327) and BlpI (nt position 9203). The Rev/RRE system was replaced by introducing the Mason-Pfizer monkey virus constitutive transport element (CTE) downstream vif (Fig. 1D).
A

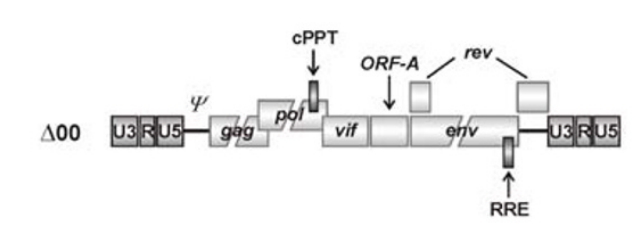

Total size

(nt)

9472

B

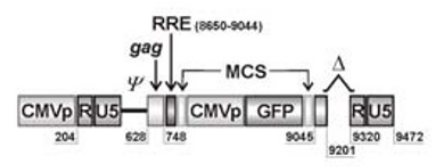

2246

2384

D LAW34

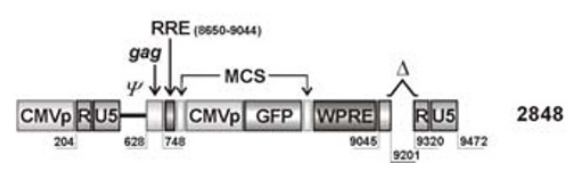

Figure 2

Schematic representation of the FIV vector constructs used. A) Parental clone $p \Delta 00$. B) Prototype LA34 vector: minimal, self-inactivating vector with both the U3 LTR domains deleted and devoid of all accessory and structural proteins except $\psi$, a 120 nt stretch at the of 5 '-end gag containing the domains important for RNA encapsidation, and the RRE. MCS, the multiple cloning site, contains the CMVP-GFP cassette used as reported gene for all vectors. C) LA34-cPPT, vector derived from LA34 by inserting the central poly-purine tract (cPPT), important for nuclear import of the FIV preintegration complex, between RRE and MCS. D) LAW34, vector obtained by inserting the woodchuck posttrascription regulatory element (WPRE) in LA34 downstream MCS. Variant vectors having a longer (310 nt) gag fragment (LA34-L and LAW34-L, respectively) were also produced but are not shown. Total size indicates the number of nucleotides of the vector without the CMVP-GFP cassette.

pGP-RRE, having the same deletion as pGP-CTE and containing RRE (nt position 8642-9067), retrieved by PCR from $\mathrm{p} \Delta 00$, in place of CTE (Fig. 1E). The Rev/RRE system was restored by providing Rev in trans.

Vector constructs

The following vector constructs were developed and tested:

LA34, produced from $\mathrm{p} \Delta 00$ by sequential steps as follows: the U3 region of the 5' LTR (nt position 1-203, as referred to $\mathrm{NC}$ 001482) was replaced with pCMV amplified from 
pcDNA3.1 plasmid (Invitrogen Life Technologies, Milan, Italy) by digestion with the restriction enzymes PshAI and SacI (New England Biolabs). To prevent LTR regeneration during reverse transcription, an internal 120 bp segment of U3 in the 3'LTR (nt position 9201-9320), containing the cis-acting transcriptional elements AP-1, AP-4 and ATF-binding sites and TATA box [20], was also removed by PCR using overlapping primers. The same strategy was applied to delete the region from nt position 749 to 9045 , encompassing most of the gag and the entire pol and env genes. Finally, the env segment containing RRE (nt position 8650-9038) was inserted downstream the gag stretch together with a multiple cloning site (MCS) containing AsuII, ClaI, SacII, BlpI, KpnI, and PacI restriction sites, and used herein for cloning the reporter GFP gene (Fig. 2B).

LA34-cPPT, obtained by inserting the central poly-purine tract (cPPT, nt position 4945-5071) that in FIV is localized within pol and is important for nuclear import of the preintegration complex (PIC) [21], in LA34. The cPPT was retrieved by PCR from $\mathrm{p} \Delta 00$ and inserted between RRE and MCS (Fig. 2C).

LAW34, obtained by inserting the woodchuck hepatitis post-transcriptional regulatory element (WPRE; kind gift of Dr. Stefano Indraccolo, University of Padua, Italy) in LA34 downstream MCS (Fig. 2D).

LA34L and LAW34L, variants of the above LA34 and LAW34, respectively, in which the gag stretch was extended from the original $120 \mathrm{nt}$ (nt position 628-747) to $310 \mathrm{nt}(628-937)$ by a two step PCR. They were prepared because reports $[22,23]$ have suggested that, in FIV, gag domains downstream the main $\psi$ determinants may contribute to efficient RNA encapsidation.

\section{Other plasmids}

The Rev provided in trans to the $p G P-R R E$ was obtained by amplifying the corresponding mRNA from RNA of FIV-Pet infected Crandell feline kidney fibroblast (CrFK) cells. The cDNA was then cloned into the pcDNA3.1 plasmid (pcDNA-Rev). Constructs pcDNA-Rev and $p G P-R R E$ were cotransfected at equimolecular ratio. FIV particles were pseudotyped with the vesicular stomatitis virus glycoprotein envelope (Env) VSV-G (495 amino acids [aa]) or the chimeric retrovirus glycoprotein RD114/TR (546 aa) [24]. VSV-G was encoded by pCMV-VSV-G derived from the pcDNA3.1 plasmid and RD114/TR by the phCMVRD114/TR plasmid (kind gift of Dr. François-Loic Cosset, Ecole Normale Supérieure, Lyon, France).

\section{Cell lines and primary murine cells}

The cells used included the CrFK, highly permissive to FIV-Pet, and the human epithelial 293T and murine fibroblast NIH-3T3, two nonfeline lines that do not per- mit FIV replication. All cells were propagated in Dulbecco's modified Eagle medium (D-MEM, Sigma-Aldrich, Milan, Italy) supplemented with $10 \%$ fetal calf serum (FCS), $100 \mathrm{U} / \mathrm{ml}$ penicillin, $100 \mu \mathrm{g} / \mathrm{ml}$ streptomycin and $2 \mathrm{mM}$ L-glutamine (Sigma-Aldrich), at $37^{\circ} \mathrm{C}$ in $5 \% \mathrm{CO}_{2}$. Murine DCs were generated from the bone marrow (BM) of 6- to 10-week old BALB/c mice. Briefly, BM cells were flushed from the femurs, filtered through a $200 \mu \mathrm{m}$ mesh to remove fibrous tissues, and cleared from erythrocytes with ammonium chloride. Residual cells were cultured at $2 \times 10^{6}$ cells $/ \mathrm{ml}$ in complete RPMI 1640 medium (SigmaAldrich), supplemented with 10\% FCS, penicillin-streptomycin and glutamine, and induced to differentiate into DCs with $20 \mathrm{ng} / \mathrm{ml}$ recombinant mouse granulocyte macrophage colony-stimulating factor (GM-CSF) and $5 \mathrm{ng} / \mathrm{ml}$ recombinant mouse interleukin (IL)-4. Floating cells were removed, and fresh GM-CSF/IL-4 enriched medium was added at days 3 and 5 of culture. On day 7, non-adherent and loosely adherent DCs were collected and analyzed by flow cytometry. While control cells cultured with no GMCSF and IL-4 were essentially negative, they were 55\% CD11c-positive, 70\% CD80-positive and 15\% CD40-positive, thus exhibiting the expression profile of immature DCs [25]. Microscopic examination of the cultures also revealed that they were rich in cells with DC morphology. Murine T lymphoblasts were produced by concanavalin A stimulation of spleen cells from the same mice. Briefly, spleen cells were cultured at $2 \times 10^{6} / \mathrm{ml}$ in 6 -well plates for 5 days in complete RPMI 1640 medium supplemented with 50 unit/ml IL-2, 10\% FCS, penicillin-streptomycin, and glutamine. These cells were $30 \%$ CD4 positive and $12 \%$ CD8 positive.

\section{Vector production}

Vectors were generated in CrFK or 293T cells. Briefly, 2.8 $\times 10^{6}$ cells were seeded in $10 \mathrm{~cm}$ Petri dishes and one day later co-transfected with one of the vector plasmids, p $\Delta$ env1, and either the VSV-G or the RD114/TR Env (4:5:1; $20 \mu \mathrm{g}$ total DNA) using a modified calcium phosphate method [26]. Transfection efficiency was evaluated 48 h later by counting GFP-positive cells by flow cytometry with a FACScan and a CELLQuest Version 2 software (BD Biosciences, Milan, Italy). Vector content in the culture fluids collected on the same day was determined by measuring FIV p25 capsid protein and the number of FIV RNA genome copies as previously described [26,27], following clarification at $1,500 \mathrm{rpm}$ for $10 \mathrm{~min}$ and $0.45 \mu \mathrm{m}$ filtration. Supernatants were aliquoted in $1 \mathrm{ml}$ volume and stored at $-80^{\circ} \mathrm{C}$ until use.

\section{Standard transduction protocol}

The day before transduction, 24-well plates were seeded with $7 \times 10^{4} 293 \mathrm{~T}$ cells, $5 \times 10^{4} \mathrm{CrFK}$ cells, $5 \times 10^{4} \mathrm{NIH} 3 \mathrm{~T} 3$ cells, $5 \times 10^{5} \mathrm{DCs}$, or $2 \times 10^{6} \mathrm{~T}$ lymphoblasts per well in 1 $\mathrm{ml}$ complete medium. Eighteen h later, the medium was 
replaced with the same volume of vector suspension. Transduction efficiency was evaluated by counting GFP positive cells by flow cytometry 2 days post-transduction (PT).

\section{FIV vector titer}

Vector titers were determined in 293T cells and expressed as number of transduction units (TU) per ml. Briefly, $7 \times$ $10^{4}$ cells were transduced as described above with the vector preparation under test serially diluted 10 -fold in culture medium. Two days later, the cells were harvested and analyzed for GFP fluorescence by flow cytometry. Each dilution was tested in triplicate.

\section{FIV vector safety evaluation}

Nucleic acids in supernatants, collected from transfected $293 \mathrm{~T}$ cells and treated as described under "Vector production", were extracted using the QIAamp Viral RNA kit (Qiagen, Milan, Italy). Genomic DNA and RNA from $6 \times$ $10^{4}$ transfected or transduced 293 T cells were extracted with QIAamp DNA Blood Kit and RNeasy kit (Qiagen), respectively. Viral and genomic RNAs were treated with RNase-free DNase (Qiagen) to eliminate residual DNA. Presence of $\mathrm{p} \Delta \mathrm{env} 1$ plasmid and RNA transcripts in supernatants and transduced cells was investigated by PCR using 295s-296as primers targeting FIV p25 capsid protein (Fig. 3A). Translocation of the U3 deletion from the 3 ' to the 5'LTR in the vector provirus was examined by amplifying genomic DNA from transduced cells with U3s-R3as primers (Fig. 3B). Inactivation of LTR mediated transcription was ascertained by amplifying cDNA of transduced cells with INs and RREas primers (Fig. 3C). Reverse transcription was carried out with an avian myeloblastosis virus reverse trascriptase (RT) (Finnzyme, Celbio, Milan Italy) and the specific antisense. Amplification profiles were as follows: initial denaturation $94^{\circ} \mathrm{C} 2$ minutes; cycling $94^{\circ} \mathrm{C} 30$ seconds, $60^{\circ} \mathrm{C}\left(54^{\circ} \mathrm{C}\right.$ for $295 \mathrm{~s}$-296as) 30 seconds, $72^{\circ} \mathrm{C} 30$ seconds (40 seconds for 295s-296as), 35 cycles; extension $72^{\circ} \mathrm{C} 10$ minutes. The $5^{\prime} \mathrm{LTR}$ amplicon was cycle sequenced using the automated ALF ExpressII DNA sequencer (GE Healthcare, Cologno Monzese, Italy).

\section{Results \\ Genome organization of the packaging and vector constructs}

Packaging and vector constructs were both derived from $\mathrm{p} \Delta 00$, a replication competent clone of FIV-Pet (Fig. $1 \mathrm{~A}$ and $2 \mathrm{~A})$.

The packaging constructs provided Gag and Pol and were under the control of the CMVp. Basically, they lacked the untranslated 5'LTR-gag region that, together with the initial part of $g a g$, form the $\psi$ signal required for viral RNA encapsidation into assembling virions and were deleted of
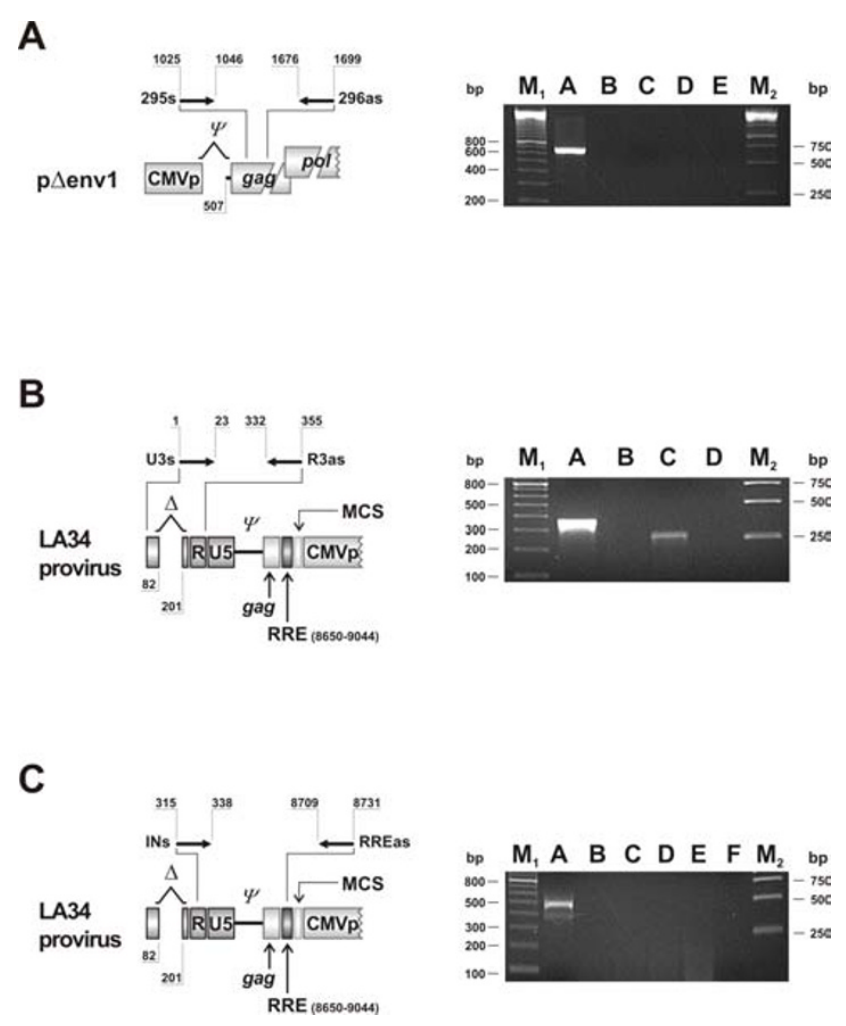

\section{Figure 3}

Safety evaluation of packaging and vector constructs. A) p $\Delta$ envl transportation from transfected to transduced cells as evaluated by gag P25 PCR using the indicated primers. Lane A: DNA from transfected 293T cells; lane B: no template control; lanes $C$ and D: DNA and RNA from transduced 293T cells; lane E: DNA from mock transfected cells.

B) Translocation of the U3 deletion to the 5'LTR, as checked by PCR using primers annealing to the beginning $U 3$ and within the $R$ region of LA34 proviral DNA. Lane A: $p \Delta 00$ plasmid (full-length LTR), lane B: no template control; lane C: DNA from transduced 293T cells, lane D: DNA from mock transduced cells. C) Analysis of LTR directed transcription as tested by PCR using primers upstream the GFP promoter. Lane A: DNA of transduced 293T cells; lane B: no template control; lanes C and D: RNA from transduced 293T cells with and without DNase treatment prior to reverse transcription; lanes E and F: RNA from mock transduced cells treated as for $C$ and $D$. Primer nt position referred to the NC 001482 sequence. $M_{1}: 100$ base-pair ladder, $M_{2}$ : Gene ruler I Kb DNA ladder (GE Healthcare).

part ( $p \Delta e n v 1$ and $p \Delta e n v 2$ constructs) or the entire ( $p G P$ $C T E$ and $p G P-R R E$ ) env. The latter constructs also lacked Vif, ORF-A, and Rev. Since nuclear export of unspliced (i.e. Gag-Pol encoding mRNA) and singly spliced mRNAs occurs through Rev binding to the RRE motif, in $p G P$-CTE the Rev/RRE system was replaced by a CTE and in $p G P$ RRE RRE was maintained and Rev provided in trans by cotransfection of pcDNA-RRE plasmid (Fig. 1B-1D). 
The SIN vector LA34 (Fig. 2B) was under the control of the CMVp and had few remnants of the FIV genome, namely 1) the $\psi$ signal, 2) the RRE motif, placed downstream $\psi$ and interacting with the Rev provided by the packaging construct or pcDNA-RRE, 3) the untranslated region between env and 3'LTR, and 4) the two LTRs, both deleted of the U3 domain to avoid the generation of functional LTRs during reverse transcription. Due to extensive rearrangement and deletions, LA34 was $2.2 \mathrm{~Kb}$ in size.

\section{Packaging and vector constructs are safe and stable}

The packaging constructs (Fig. 1B-1E) were developed and tested for stability and safety (non-infectivity) in CrFK and 293T cells. Briefly, the packaging constructs were transfected into cells which were propagated for at least two weeks and monitored twice a week for FIV p25 and viral RNA release into supernatant. Further, cell-free supernatants were collected at 7 and 14 days post-transfection, and seeded into fresh CrFK cell that were cultivated for additional two weeks. Except for a low and transient production of p 25 found two-three days posttransfection, neither FIV RNA nor infectious particles were found in supernatants (data not shown). The results indicated that the packaging constructs were free from residual $\mathrm{p} \Delta 00$ plasmid molecules, were stable, and did not generate infectious virus.

Transduction efficiency, stability, and safety were tested in CrFK and 293T cells, using LA34 pseudotyped with VSV-G (LA34/VSV-G) generated in CrFK or 293T cells with $\mathrm{p} \Delta$ env1 as packaging construct. The proportions of GFPpositive CrFK and 293T cells 2 days post-transfection were generally greater than $75 \%$. As determined by measuring p25 and number of vector RNA copies in the supernatant from day 2 to 7 post-transfection, LA34/VSV-G production peaked at day 2 or 3 (data not shown). Vector particles collected at these times were pelleted by ultracentrifugation and analyzed by western blot for protein content. Regardless of whether produced in CrFK or $293 \mathrm{~T}$ cells, the vector generated protein patterns that, with the obvious exception of Env, were identical to the one of whole FIV-Pet virus used as control, indicating that proper generation and maturation of virions had taken place (data not shown). As shown in Table 1, vector titers exceeded $10^{9} \mathrm{RNA}$ copies $/ \mathrm{ml}$ and $10^{6} \mathrm{TU} / \mathrm{ml}$ in $293 \mathrm{~T}$ cells and were slightly higher when the vector was produced in CrFK rather than 293T cells.

Vector safety was evaluated by several approaches. First, the progeny particles were checked for $\mathrm{p} \Delta \mathrm{env} 1$ incorporation by testing them as well as transduced cells for a gag p25 sequence contained in the packaging construct only (Fig. 1B to $1 \mathrm{E}$ ). The $674 \mathrm{bp}$ amplicon generated by the PCR and RT-PCR assays used was readily detected in the DNA of transfected cells (positive control) but uniformly absent in the vector particles (not shown) and in transduced cells (Fig. 3A). Second, it was checked whether the U3 deletion, created in the 3'LTR of the vector construct, was indeed translocated to the 5'LTR of proviral DNA by using primers that generated amplicons of different sizes from the full-length (352 bp) and the U3 deleted LTRs (232 bp). The amplicon generated from the DNA of transduced cells was clearly smaller than the one generated from the $\mathrm{p} \Delta 00$ plasmid used as a source of full-length LTR (Fig. 3B) and had the sequence expected for the deleted LTR (not shown). Third, functional inactivation of the $5^{\prime}$ LTR was checked by examining transduced cells for LA34 RNA genomes, the transcription of which would have required a full-length 5'LTR. As shown by Fig. 3C, while the DNA of transduced cells was clearly positive for LA34 sequences, the RNA obtained from the same cells was uniformly negative, regardless of whether it was digested or not with RNase-free DNase. Collectively, these findings demonstrated that LA34 is indeed a SIN vector, that the pseudotyped particles it generates are safe, and that no vector RNA is produced by LA34 transduced cells.

\section{LA34 is best packaged by $p \Delta$ env l and preferentially transduces feline cells}

The packaging constructs were tested for ability to produce LA34/VSV-G virions in 293T cells that were transfected with equimolecular amounts of packaging, vector, and VSV-G plasmids. Supernatants collected 2 days posttransfection were analyzed for virus release by measuring p25 and vector RNA genome copies and for competence for transduction in 293T cells. The efficiency of transfection was similar for all plasmid combinations and averaged $80 \%$. As shown in Table 2, LA34/VSV-G production with $\mathrm{p} \Delta \mathrm{env} 1$ and $\mathrm{p} \Delta \mathrm{env} 2$ was very high, as shown by the high levels of p25 produced, $10^{9}$ vector RNA copies and $10^{6} \mathrm{TU} / \mathrm{ml}$. In contrast, both vector RNA and TU titers of VSV-G pseudotyped particles produced by using pGP-RRE and pGP-CTE were one and two logs lower, respectively, suggesting that virus release, rather than infectivity, was less efficient. The pseudotyped particles generated with $\mathrm{p} \Delta \mathrm{env} 1$ and $\mathrm{p} \Delta \mathrm{env} 2$ were further tested for transduction in CrFK. Since the LA34/VSV-G generated with p $\Delta$ env1 performed slightly better in this cell subtype, this construct was selected as packaging for subsequent experiments (data not shown).

Transduction efficiency and duration of transgene expression were assessed by inoculating LA34/VSV-G produced in $293 \mathrm{~T}$ cells into CrFK, 293T and NIH-3T3 cultures at varying RNA copy numbers. The best results were obtained with $10^{9}$ copies, corresponding roughly to $10 \mathrm{TU} / \mathrm{cell}$, which at the first readout, 4 days PT, transduced $66 \%$ and $52 \%$ of CrFK and $293 \mathrm{~T}$ cells, respectively. In contrast, GFP-positive NIH-3T3 cells rarely exceeded $20 \%$, indicating that these cells were largely refractory to transduction 
Table I: Vector titers generated from transfected 293T or CrFK cells ${ }^{\mathrm{a}}$

\begin{tabular}{lcccc}
\hline $\begin{array}{l}\text { Vector/Env used for } \\
\text { pseudotyping }\end{array}$ & Vector produced in & $\begin{array}{c}\text { Vector titer (RNA } \\
\text { copies/ml) }\end{array}$ & Concentration & Transduction units/ml $^{\mathrm{b}}$ \\
\hline LA34/VSV-G & CrFK & $8.5 \times 10^{9}$ & None & $3.8 \times 10^{6}$ \\
LAW34/VSV-G & $293 \mathrm{~T}$ & $1.8 \times 10^{9}$ & None & $1.2 \times 10^{6}$ \\
& $293 \mathrm{~T}$ & $5.0 \times 10^{9, \mathrm{c}}$ & None & $2.5 \times 10^{7}$ \\
& & $2.1 \times 10^{10, \mathrm{c}}$ & Ultracentrifugation & $3.0 \times 10^{7}$ \\
LAW34/RDII4/TR & $293 \mathrm{~T}$ & $7.0 \times 10^{9}$ & PBd & $3.0 \times 10^{7}$ \\
& $293 \mathrm{~T}$ & $1.4 \times 10^{10, \mathrm{c}}$ & Ultracentrifugation & $6.0 \times 10^{7}$ \\
\end{tabular}

a Titers measured in supernatants collected 2 days post-transfection, average of three independent experiments.

b Measured in 293T cells.

c Same supernatant, titrated as such or after 10 fold concentration by ultracentrifugation.

d Polybrene.

by this vector. Furthermore, during 25 days of observation, GFP-positive cell numbers remained essentially unchanged regardless of initial transduction level, indicating that transduction and transgene expression were stable, as a likely result of vector integration into the cell genomes (Fig. 4A). No infectious virus was detected in the transduced cultures at any time, not even after passaging the culture fluids in fresh CrFK cells repeatedly (data not shown).

\section{Insertion of the post-transcription regulatory element WPRE increases LA34 transduction efficiency for NIH-3T3} Because the findings above were indicative of a preferential ability of LA34 to transduce feline CrFK cells relative to the non feline cells 293T and NIH-3T3, we made efforts to widen its breadth of action by improving nuclear translocation of PIC, stabilization of transgene mRNAs, and incorporation of vector RNA into pseudotyped particles that are major determinants of lentiviral cell transduction and transgene expression [28]. Two short single-stranded regions of the lentiviral genome DNA (flaps) are believed to optimize viral genome folding, thus enhancing its steric fit in the nuclear pore [29]. In FIV, one of these flaps is located upstream the 3' LTR (U3PPT) and the other close to the 3' end of pol (central PPT, cPPT) [21]. Since LA34 contains only the U3PPT, we inserted the CPPT between RRE and MCS (construct LA34-CPPT, Fig. 2C), similar to what already done in the FIV vectors developed in previous reports $[28,30]$. However, the change had no appreciable effects on vector performance in any of the three cell lines (Fig. 3B).

When we inserted the RNA transport WPRE element downstream of MCS in LA34 (LAW34, Fig. 2D), so that it could be incorporated into transgene mRNA, the efficiency of transduction improved greatly. Compared to LA34, LAW34 showed similar RNA titers but the TUs per $\mathrm{ml}$ were approximately $1 \log$ higher (Table 1$)$. Most importantly, LAW34/VSV-G gave rates of NIH-3T3 cell transduction that were nearly twice as great. In fact, close to $50 \%$ of the latter cells expressed GFP and did so for at least 4 weeks (Fig. 4B and data not shown).

Recent reports have suggested that the main packaging domain in the gag of FIV is comprised in a $120 \mathrm{nt}$ stretch [31]; however, previous studies had suggested the existence of additional encapsidation determinants in gag, bringing $\psi$ to about $300 \mathrm{nt}$ in size [23]. We, thus, also constructed versions of LA34 and LAW34 having a $311 \mathrm{nt}-$ long $\psi$ (LA34-L and LAW34-L). When compared to the respective vectors with 120 nt-long $\psi$, these versions showed no appreciably increased titers (data not shown) and, with the exception of LAW34-L for CrFK cells, had reduced transduction efficiencies (Fig. 4C). As a result of these studies, LAW34 was selected for the experiments below.

\section{Effect of transduction protocol on LAW34 efficiency}

In these experiments, we compared the standard transduction method described under Methods with several protocols that have been shown to increase transduction efficiency by other vectors. These included: 1) vector ultracentrifugation, a procedure frequently used to concentrate VSV-G pseudotyped viruses that, unlike lentiviral Env-

Table 2: LA34/VSV-G pseudotyped particles generated 2 days post-transfection in $293 T$ cells $^{a}$

\begin{tabular}{|c|c|c|c|}
\hline $\begin{array}{l}\text { Packaging } \\
\text { construct } \\
\text { used for } \\
\text { vector } \\
\text { production }\end{array}$ & $\begin{array}{l}\text { p25 optical } \\
\text { density }\end{array}$ & $\begin{array}{c}\text { Vector RNA } \\
\text { copies/ml }\end{array}$ & $\begin{array}{l}\text { Transduction } \\
\text { units } / \mathrm{ml}^{\mathrm{b}}\end{array}$ \\
\hline pDenvl & 2.24 & $9.3 \times 10^{9}$ & $6.4 \times 10^{6}$ \\
\hline p Denv2 & $>2.50$ & $3.0 \times 10^{9}$ & $3.4 \times 10^{6}$ \\
\hline pGP-CTE & 1.09 & $2 \times 10^{6}$ & $1.0 \times 10^{3}$ \\
\hline pGP-RREc & 1.92 & $8.1 \times 10^{8}$ & $3.0 \times 10^{5}$ \\
\hline
\end{tabular}

a Average of three independent experiments, mean efficiency of transfection $80 \%$ (range $75-90 \%$ );

b Titer measured in the indicated cells 2 days post-transduction;

c Rev provided in trans by cotransfection of pcDNA-Rev. 
A

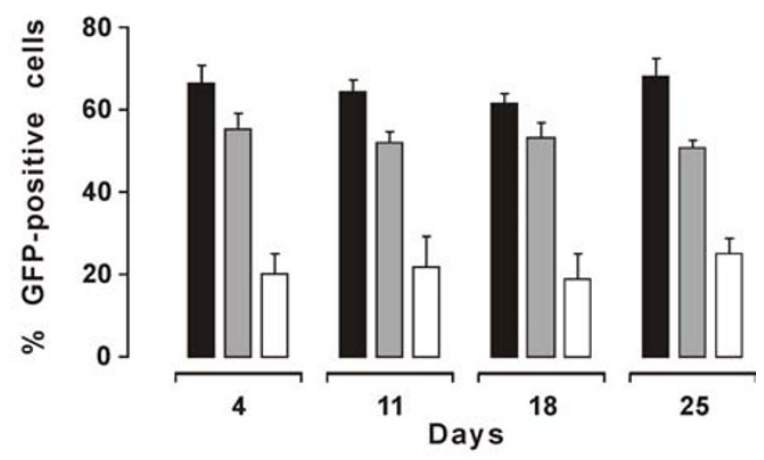

B
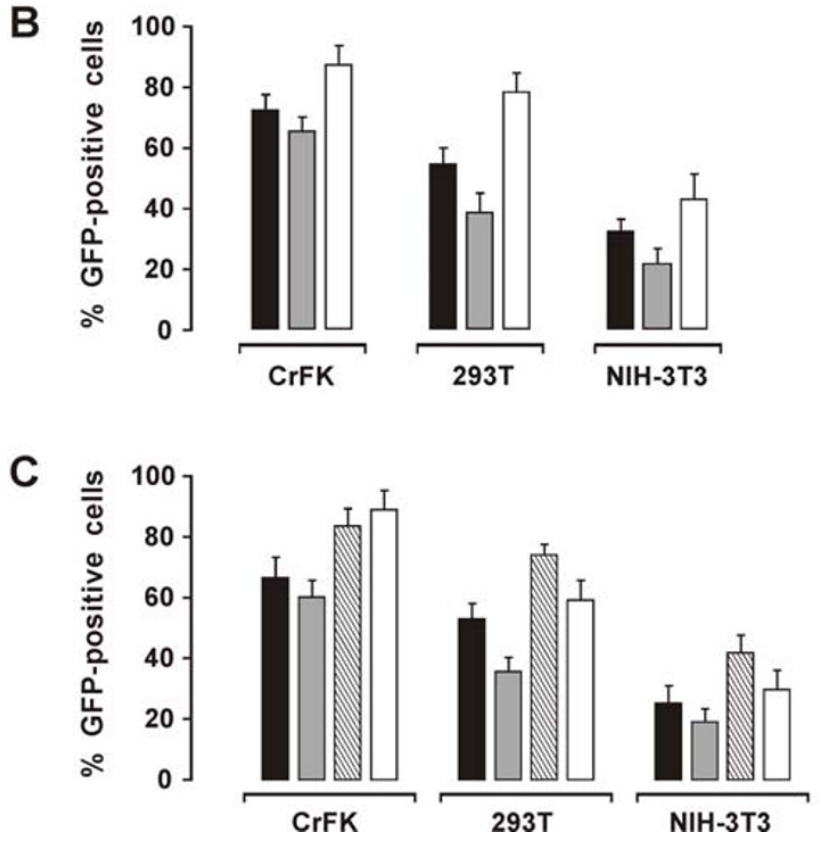

Figure 4

Efficiency and duration of transduction by naïve and variously modified LA34/VSV-G as determined in CrFK, 293T and NIH-3T3 cells. A) Efficiency and stability of transduction in CrFK (solid columns), 293T (shaded columns) and NIH-3T3 cells (empty columns) as evaluated by flow cytometry at the indicated times PT. B) LA34-cPPT (shaded columns), LAW34 (empty columns) and LA34 (solid columns). Percent GFP-positive cells evaluated by flow cytometry 4 days PT. C) LA34-L (shaded columns) and LAW34-L (empty columns) versus LA34 (solid columns) and LAW34 (striped columns). Percent GFP-positive cells evaluated by flow cytometry 4 days PT. Bars represent the standard deviation as calculated from three independent experiments.

coated pseudoparticles, do not tend to shed Env [32]; 2) low-speed centrifugation following poly-L-lysine addition [33]; 3) addition of polybrene (PB), a polycation that forms large complexes with viral particles leaving out nonviral proteins and other factors that may be inhibitory (protocol PB) [34]; 4) treating the cells with the vector twice, four h apart (double transduction; protocol DT); and 5) combined use of 3 and 4 (protocol PB/DT). LAW34/VSV-G produced in $293 \mathrm{~T}$ cells $\left(5 \times 10^{9}\right.$ vector RNA copies/ml) was concentrated 10 -fold by ultracentrifugation at $200,000 \mathrm{~g}$ at $4^{\circ} \mathrm{C}$ for $2 \mathrm{~h}$. In spite of a 4 -fold increment in vector RNA titer, transduction efficiency was essentially unchanged relative to the standard method (Table 1). The same output was observed by using protocol 2 in which the virus was concentrated by aggregation with poly-L-lysine. Moreover, the use of poly-L-lysine often caused shrinking, granulation, and, occasional detachment of the cells (data not shown). Protocols 3 to 5 were instead beneficial. A dose-response study of $\mathrm{PB}$ in $293 \mathrm{~T}$ using a vector RNA copy/cell ratio of 200/1 cells (approx. $10 \mathrm{TU} /$ cell) yielded the highest transduction rates at $8 \mu \mathrm{g} / \mathrm{ml}$ (Fig. 5A). Importantly, at this dose PB more than doubled the proportion of transduced NIH3 T3 cells (Fig. 5B), suggesting that this protocol substantially increased virus infectivity on this cell type. At same conditions, protocol DT also increased transduction of all cell types (data not shown). However, protocol DT/PB was the most effective, since transduced cells exceeded $90 \%$ at day 4 (Fig 5B). Overall, the results also underlined the robustness of LAW34 under various test conditions.

\section{LA34 pseudotyped with RDI I 4/TR transduces NIH-3T3 cells efficiently}

LAW34 was pseudotyped with RD114/TR in 293T cells, and the vector thus produced (LAW34/RD114/TR; Fig. 6A) was titrated for TU in the same cell substrate using the ultracentrifugation and the $\mathrm{PB} / \mathrm{DT}$ protocols. Again, in spite of a three-fold increment of the vector RNA titer after supernatant ultracentrifugation, transduction efficiency was lower compared to the PB protocol, confirming the modest performances of ultracentrifugation in our hands (Table 1). LAW34/RD114/TR and LAW34/VSV-G had essentially same number of vector RNA copies $/ \mathrm{ml}$, yet the former exhibited a 4 fold higher $293 \mathrm{~T}$ transduction titer when complexed with $\mathrm{PB}$, confirming the efficacy of this protocol even in the case of easy-to-transduce cells.

LAW34/RD114/TR and LAW34/VSV-G were also compared for transduction efficiency using the standard protocol, i.e. with no further manipulations or additions. Supernatants of packaging $293 \mathrm{~T}$ cells diluted to achieve a vector RNA copy/cell ratio 1/200 of either vector performed equally in $293 \mathrm{~T}$ and CrFK cells even after prolonged propagation (Fig. 6B and data not shown). In contrast, in NIH-3T3 cells LAW34/RD114/TR transduced with an efficiency the VSV-G counterpart had exhibited only when used with the PB protocol (Fig. 5B and 6B). Thus, LAW34/RD114/TR proved effective at transducing 
A

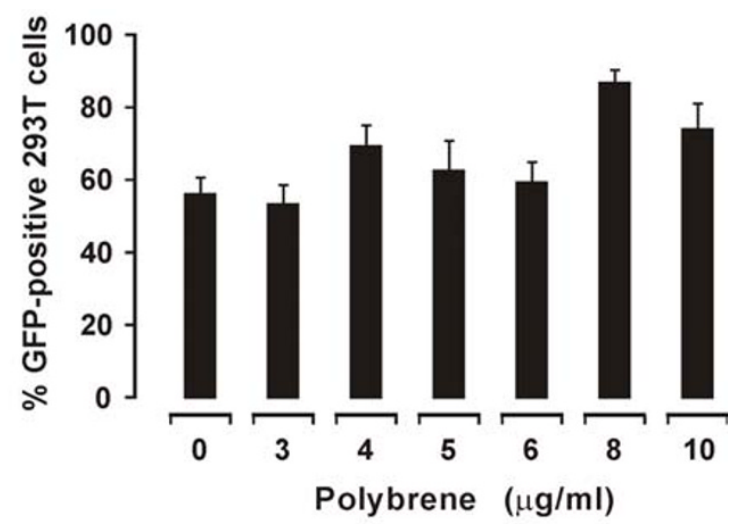

B

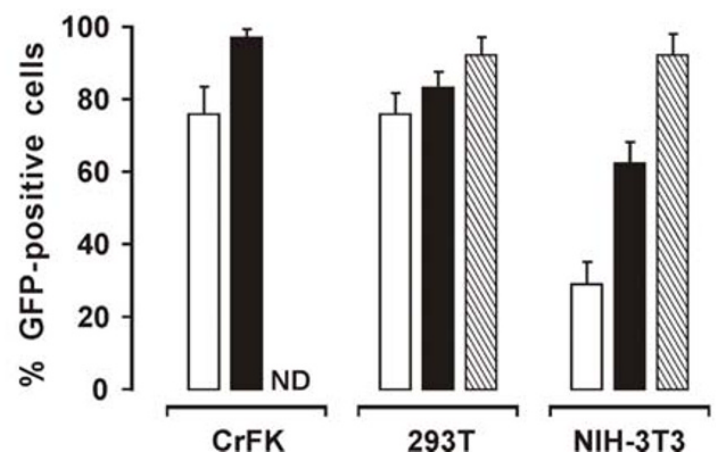

Figure 5

Evaluation of three transduction protocols. A) Effect of PB at the indicated concentrations on LA34/VSV-G transduction of 293T cells. B) Effect of using the standard (empty columns), PB (solid columns) or PB/DT protocol (striped columns) on LA34/VSV-G transduction of the indicated cells. Bars and percent GFP-positive cells evaluated as in Fig. 4. ND, not done.

non feline cell lines with no need for treatments known to boost transduction efficiency.

\section{LAW34 transduces murine DCs and T lymphocytes efficiently}

BM-derived DCs were transduced with VSV-G or RD114/ TR pseudotyped LAW34 by using 200 vector RNA copies per cell and protocols PB, DT, and DT/PB. Cells were examined 2 days later for GFP expression. LAW34RD114/TR transduced much more efficiently than LAW34/VSV-G, regardless of protocol used. This striking difference, already clearly evident by microscopic inspection, was confirmed by flow cytometry analysis of the cells: whereas the fluorescence signal of LAW34/VSV-G transduced cells was barely distinguishable from that of mock transduced cells, LAW34/RD114/TR produced a clearly defined peak at $10^{1} \mathrm{FL} 1-\mathrm{H}$, indicating that most DCs were transduced and actively expressing GFP (Fig. 7A). In fact, LAW34/RD114/TR performed better with all 3 protocols, transducing up to $52 \%$ DCs when the DT/PB

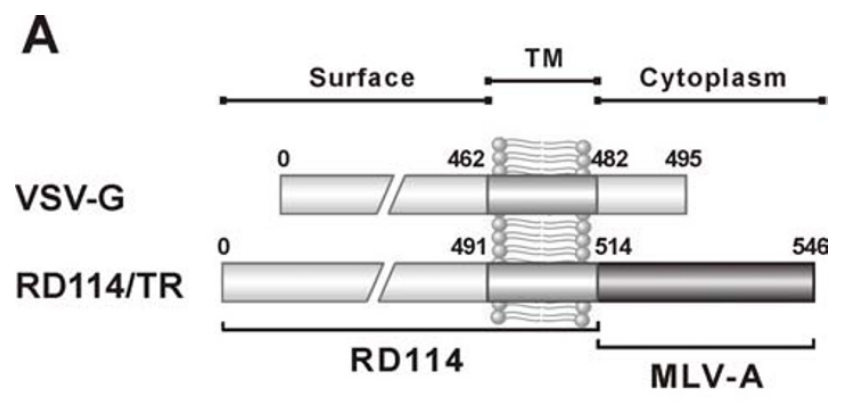

B

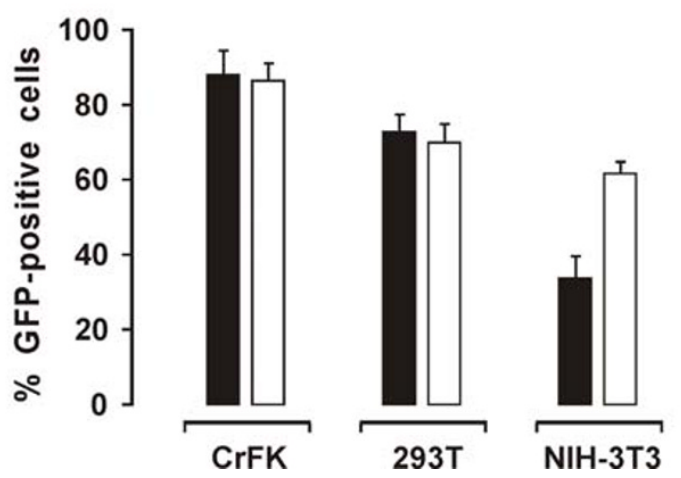

Figure 6

Effect of pseudotyping LAW34 with different Env. A) Diagrammatic representation of the VSV-G and RDI I4/TR glycoproteins. The latter has the extracellular and transmembrane domains of the feline endogenous retrovirus RDII 4 and the cytoplasmic tail of an amphotropic murine leukemia virus [36]. Numbers are aa residues. B. Transduction efficiency for the indicated cell types of LAW34 pseudotyped with VSV-G (solid columns) or RDI I4/TR Env (empty columns). Bars and percent GFP-positive cells evaluated as in Fig. 4.

protocol was used versus $17 \%$ with LAW/VSV-G (Fig. 7B and 7C). LAW34/RD114/TR transduction was also examined for possible effects on markers expression by DCs. DCs transduced with LAW34/RD114/TR at 200 vector RNA copies per cell using the PB protocol were compared to similarly treated cells except that the vector was replaced by the supernatant of mock-transfected cells. Relative to mock treated DCs, transduced DCs showed no changes in CD11c and CD80 expression but underwent a substantial increase of CD40-positive cells which was already evident by day 2 PT (Fig. 7D) and lasted throughout the observation period of 10 days (not shown). Of note, GFP expression by transduced cells, monitored in parallel, increased slightly over time (data not shown). 
A

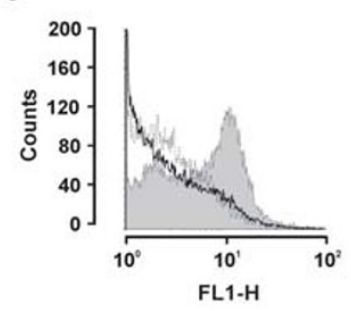

C

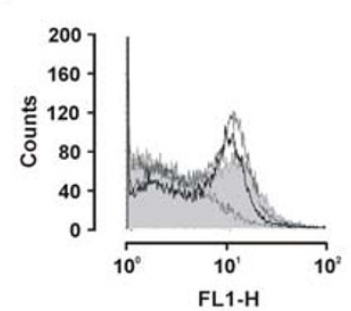

E

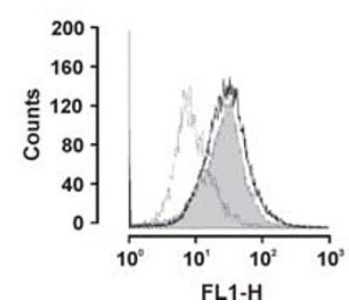

B

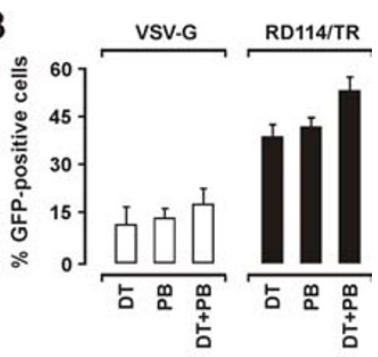

D

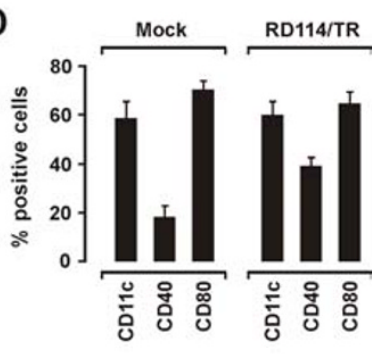

$\mathbf{F}$

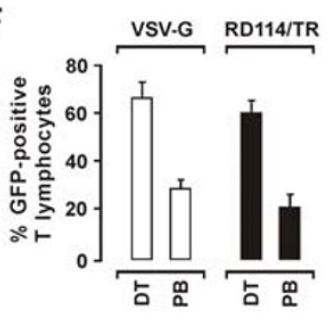

\section{Figure 7}

Transduction of primary murine DCs and T lymphocytes with LAW34/VSV-G and LAW34/RDI I4/

TR. A) Flow cytometry analysis of DCs transduced with LAW34/VSV-G (thick line) or LAW34/RD I I4/TR (grey area) using PB protocol. Dotted line, untransduced, PB-treated cells. B) Efficiency of DC transduction by LAW34/VSV-G and LAW34/RDI I 4/TR using the 3 protocols indicated. Percent GFP positive cells evaluated by flow cytometry 2 days PT. C) Intensity of GFP expression in the LAW34/RDI I4/TR transduced DCs in panel B, using protocols DT (thick line), PB (grey area), and DT/PB (grey line). Dotted line, untransduced DCs. D) Specific markers in DCs transduced with LAW34/ RDI I 4/TR using protocol PB or mock transduced, as examined 2 days PT. E) Flow cytometry analysis of T lymphocytes derived from murine spleen cells by ConA/IL-2 stimulation and transduced with LAW34/VSV-G (thick line) or LAW34/ RDI I4/TR (grey area) using DT protocol. Dotted line, untransduced T lymphocytes. F) Efficiency of T lymphocyte transduction by LAW34/VSV-G and LAW34/RDI I4/TR using the protocols indicated.

LAW34/VSV-G or LAW34/RD114/TR were also compared for ability to transduce cultured murine $\mathrm{T}$ lymphoblasts using the $\mathrm{PB}$ or the DT protocol and same vector/cell ratios. As shown by Fig. 7E and 7F, which reports the readings at day 2 PT, GFP positive cells ranged around 60\%

and fluorescence peaks were superimposible regardless of the Env used for pseudotyping. Also, the use of PB greatly reduced the efficiency of $T$ lymphoblast transduction by both LAW34/VSV-G and LAW34-RD114/TR, in spite that no obvious effects on cell viability were noted. Proportions of $\mathrm{CD} 4$ and $\mathrm{CD} 8$ positive cells in the cultures remained stable for at least 10 days, following transduction (data not shown).

\section{Discussion}

Lentivirus-derived vectors possess several advantages, including that they ensure stable and tightly controlled expression of transgenes by integrating into the cell genome, integrate preferentially into actively transcribed genes yet distantly from cellular promoters [35-37], transduce quiescent and dividing cells alike $[7,30,38]$, and possibly have a lower insertional mutagenesis risk relative to vectors derived from other retroviruses [39]. Among such vectors, those derived from FIV have been shown to be as efficient as HIV vectors at transducing a variety of cell types and tissue compartments in vivo and have the added advantage of posing less safety concerns $[12,28]$. However, the FIV vectors described to date performed poorly when used for transducing immune cells [14-16,40], a limitation that prompted us to develop an FIV vector that might efficiently and stably deliver genes into DCs and T cells.

The vector we first constructed, LA34 is entirely derived from an FIV strain known to be much attenuated compared to field isolates $[17,30]$ and, to further increase its safety, is self-inactivated by bearing LTRs partially deleted and totally inactive. The expression construct could be easily pseudotyped with two distinct Envs that conferred either a broad or a more restricted cell tropism. With the aim to obtain a vector that could be used in mouse models, efficiency at transducing the murine cell line NIH-3T3 was a major guiding criterion in its design as well as in optimizing transduction protocol. However, in its original format LA34 performed poorly with NIH-3T3 cells. Thus, in the attempt to overcome this drawback, the following modifications were introduced:

1) lentiviruses have evolved a PIC consisting of cellular and viral proteins which effectively delivers viral cDNA in close proximity of the cell genome. Since LA34 lacked CPPT, one of the two single-strand flaps generated during reverse transcription that are thought to optimize cDNA folding and enhance its steric fit in the nuclear pore [29], we inserted it between RRE and expression cassette. In contrast to what observed with other FIV vector formats $[28,30]$, this modification failed to improve LA34 performances. The reason(s) was not addressed, but it is plausible that p34TF10, the parental clone from which LA34 was produced, is per se minimally dependent on this motif 
or that the p34TF10-derived cPPT we used, being slightly different in sequence from the ones previously used [30], was poorly functional.

2) the genome of lentiviruses is encapsidated through the mutual recognition of specific RNA sequences $(\psi)$ and specific viral and cellular proteins [41]. In FIV, $\psi$ is believed to consist of at least two discontinuous core regions, the first located upstream the major splice donor site, the second extending into gag. While later analyses have located the principal $\psi$ domains within the first 100$120 \mathrm{nt}$ [31], in early RNA protection experiments, optimal viral RNA encapsidation was obtained by extending the gag region to $311 \mathrm{nt}$ [23]. A 310-nt sequence, that in our FIV encompasses the entire region found to be important in the above study [23], was then substituted for the 120nt sequence present in LA34, but this brought no benefits and actually impaired nonfeline cell transduction. Again, the reason(s) for this discrepancy relative to previous studies was not investigated but it might lie in differences between the FIV clones used or in the genomic organization of the vectors developed. In any case, these results, together with the reported presence of negative regulatory sequences upstream and within $\psi[31,42]$ and the consideration that fewer parental virus sequences are present in a vector the better, made us to decide in favor of the vector with the shorter $\psi$.

3) the introduction of the RNA transport element WPRE into FIV- and HIV-derived vectors has been shown to stabilize and enhance transgene expression $[30,43]$. When we introduced this element into LA34 we also observed a favorable impact on vector performance: the vector thus modified (LAW34) not only showed higher titers relative to LA34 but also transduced with enhanced efficiency all the cell lines. Importantly, the positive effects of WPRE were most evident in NIH-3T3 cells, suggesting that LAW34 might be particularly suitable for transducing murine cells.

We then focused on further optimizing LAW34 for usage with murine NIH-3T3 cells by modifying external coat and transduction protocol, two factors known to be as important as the vector itself for successful gene targeting and delivery. VSV-G pseudotyping is extremely convenient for vector development and in vitro testing since, being the cell receptor for VSV a ubiquitous phospholipid [44], it allows vector access into virtually any cell type, but this extended host range can be unpractical for vector usage in vivo where restricted cell targeting is often required. Moreover, VSV-G pseudotyped vectors have proved toxic for ex vivo cells and prone to inactivation by complement [45]. Thus, while in setting up the vector we had used VSV-G pseudotyped particles, in subsequent studies, among the many Env glycoproteins available for pseudotyping retrovirus vectors particles [46], we selected RD114/TR, derived from the feline endogenous ecotropic virus RD114, which has a sodium-dependent neutralamino-acid transporter as cellular receptor, but having the cytoplasmatic tail of MLV-A. This chimeric Env, which in other systems has been shown to substantially increase vector titers, improve cellular localization and interaction with Gag [47], resist lysis by human complement and permit transduction of primary human lymphocytes and stem cells [24], to our knowledge had never been used with FIV vectors. The choice proved fortunate, because LAW34 pseudotyped with this Env (LAW34/RD114/TR) transduced at high efficiency all the cell lines tested and compared favorably to VSV-G pseudotyping regardless of the transducing protocol used.

We finally examined the efficiency of LAW34 at transducing ex vivo murine DCs and T cells using different transduction protocols. The results showed that the vector has the ability to efficiently transduce both cell types, but that the Env with which it is pseudotyped is critical for DC transduction. Indeed, while T cells were transduced to similar extents regardless of whether the vector was coated with VSV-G or RD114/TR, DCs were transduced by LAW34/RD114/TR alone, thus suggesting that previous unsatisfactorily attempts to efficiently transduce DCs with FIV vectors $[14-16,40]$ may have been due to the use of Envs that did not permit proper entry into these cells. Of note, in agreement with findings showing that DCs transduced with different vectors develop a mature phenotype $[1,6,11]$, murine DCs transduced with LAW34/RD114/TR exhibited at least some of the morphological and surface markers known to accompany DC maturation, thus showing that they were still functional. A further observation of interest was that LAW34 transduction of DCs and T cells occurred also in the absence of added facilitating agents, such as $\mathrm{PB}$, that preferably should be avoided when vectors are used in vivo.

\section{Conclusion}

The FIV-derived vector LAW34 has features compatible with a prospective usage for vaccinal purposes. These include the small size of the vector that permits to accommodate large transgenes, possibly encoding more than one protein, and the ability to deliver such transgenes into two key cell types of the immune system. In vitro and in vivo studies investigating whether transduced DCs can serve as antigen presenting cells and whether transduced $\mathrm{T}$ cells can be used in prime-boost vaccine experiments are currently underway.

\section{Competing interests}

The author(s) declare that they have no competing interests. 


\section{Authors' contributions}

All Authors read and approved the final manuscript. MP is the project leader and wrote the paper, LV and AR constructed the vector and made substantial contribution to its design, FB designed and constructed the packaging vectors, FC and BDS were involved in the in vitro characterization of the vector, GF produced and analyzed the dendritic cells, $\mathrm{MB}$ was involved in drafting and critical revising the manuscript.

\section{Acknowledgements}

This study was supported by Ministero dell'Istruzione, dell'Università e della Ricerca, grants FIRB 200I e PRIN 2005, and Ministero della Salute Istituto Superiore di Sanità, "VI ${ }^{\circ}$ Programma Nazionale di Ricerca sull'AIDS 2006" "Progetto Nazionale AIDS - ICAV".

\section{References}

I. Figdor CG, de Vries IJ, Lesterhuis WJ, Melief CJ: Dendritic cell immunotherapy: mapping the way. Nat Med 2004, 10:475-480.

2. Randolph GJ, Angeli $V$, Swartz MA: Dendritic-cell trafficking to lymph nodes through lymphatic vessels. Nat Rev Immunol 2005, 5:617-628.

3. Marathe JG, Wooley DP: Is gene therapy a good therapeutic approach for HIV-positive patients? Genet Vaccines Ther 2007, 5:5.

4. Palucka AK, Laupeze B, Aspord C, Saito H, Jego G, Fay J, Paczesny S, Pascual V, Banchereau J: Immunotherapy via dendritic cells. Adv Exp Med Biol 2005, 560: 105-II4.

5. Wu Q, Xia D, Carlsen S, Xiang J: Adenovirus-mediated transgene-engineered dendritic cell vaccine of cancer. Curr Gene Ther 2005, 5:237-247.

$6 . \quad J o o s s \mathrm{~K}$, Chirmule N: Immunity to adenovirus and adeno-associated viral vectors: implications for gene therapy. Gene Ther 2003, 10:955-963.

7. Kafri T: Gene delivery by lentivirus vectors an overview. Methods Mol Biol 2004, 246:367-390.

8. Buffa V, Negri DR, Leone P, Borghi M, Bona R, Michelini Z, Compagnoni D, Sgadari C, Ensoli B, Cara A: Evaluation of a self-inactivating lentiviral vector expressing simian immunodeficiency virus gag for induction of specific immune responses in vitro and in vivo. Viral Immunol 2006, 19:690-70I.

9. Chinnasamy N, Treisman JS, Oaks MK, Hanson JP, Chinnasamy D: Ex vivo generation of genetically modified dendritic cells for immunotherapy: implications of lymphocyte contamination. Gene Ther 2005, 12:259-27|.

10. He Y, Zhang J, Mi Z, Robbins P, Falo LD Jr: Immunization with lentiviral vector-transduced dendritic cells induces strong and long-lasting $\mathbf{T}$ cell responses and therapeutic immunity. J Immunol 2005, 174:3808-38I7.

II. Kung SK, Bonifacino A, Metzger ME, Ringpis GE, Donahue RE, Chen IS: Lentiviral vector-transduced dendritic cells induce specific $T$ cell response in a nonhuman primate model. Hum Gene Ther 2005, 16:527-532.

12. Romano G: Current development of lentiviral-mediated gene transfer. Drug News Perspect 2005, I 8: 128-134.

13. Sparger EE: FIV as a model for HIV: an overview. In In vivo Models of HIV Infection and Control Edited by: Friedman H, Specter S. Bendinelli. New York: Springer; 2006: 149-237.

14. Curran MA, Kaiser SM, Achacoso PL, Nolan GP: Efficient transduction of nondividing cells by optimized feline immunodeficiency virus vectors. Mol Ther 2000, I:3I-38.

15. Poeschla EM, Wong-Staal F, Looney DJ: Efficient transduction of nondividing human cells by feline immunodeficiency virus lentiviral vectors. Nat Med 1998, 4:354-357.

16. Price MA, Case SS, Carbonaro DA, Yu XJ, Petersen D, Sabo KM, Curran MA, Engel BC, Margarian H, Abkowitz JL, et al.: Expression from second-generation feline immunodeficiency virus vectors is impaired in human hematopoietic cells. Mol Ther 2002, 6:645-652

17. Pistello M, Moscardini M, Mazzetti P, Bonci F, Zaccaro L, Isola P, Freer G, Specter S, Matteucci D, Bendinelli M: Development of feline immunodeficiency virus ORF-A (tat) mutants: in vitro and in vivo characterization. Virology 2002, 298:84-95.

18. de Parseval A, Elder JH: Demonstration that orf2 encodes the feline immunodeficiency virus transactivating (Tat) protein and characterization of a unique gene product with partial rev activity. J Virol 1999, 73:608-617.

19. Mustafa F, Jayanth P, Phillip PS, Ghazawi A, Schmidt RD, Lew KA, Rizvi TA: Relative activity of the feline immunodeficiency virus promoter in feline and primate cell lines. Microbes Infect 2005, 7:233-239.

20. Bigornia L, Lockridge KM, Sparger EE: Construction and in vitro characterization of attenuated feline immunodeficiency virus long terminal repeat mutant viruses. J Virol 200I, 75: $1054-1060$.

21. Whitwam T, Peretz M, Poeschla E: Identification of a central DNA flap in feline immunodeficiency virus. J Virol 200I, 75:9407-94I4.

22. Browning MT, Mustafa F, Schmidt RD, Lew KA, Rizvi TA: Sequences within the gag gene of feline immunodeficiency virus (FIV) are important for efficient RNA encapsidation. Virus Res 2003 , 93:199-209.

23. Kemler I, Barraza R, Poeschla EM: Mapping the encapsidation determinants of feline immunodeficiency virus. J Virol 2002, 76: I 1889-11903

24. Sandrin V, Boson B, Salmon P, Gay W, Negre D, Le Grand R, Trono $D$, Cosset FL: Lentiviral vectors pseudotyped with a modified RDII4 envelope glycoprotein show increased stability in sera and augmented transduction of primary lymphocytes and CD34+ cells derived from human and nonhuman primates. Blood 2002, 100:823-832.

25. Munz C, Steinman RM, Fujii S: Dendritic cell maturation by innate lymphocytes: coordinated stimulation of innate and adaptive immunity. J Exp Med 2005, 202:203-207.

26. Pistello M, Bonci F, Flynn JN, Mazzetti P, Isola P, Zabogli E, Camerin $V$, Matteucci $D$, Freer $G$, Pelosi P, Bendinelli M: AIDS vaccination studies with an ex vivo feline immunodeficiency virus model: analysis of the accessory ORF-A protein and DNA as protective immunogens. J Virol 2006, 80:8856-8868.

27. Matteucci D, Pistello M, Mazzetti P, Giannecchini S, Isola P, Merico A, Zaccaro $L$, Rizzuti $A$, Bendinelli M: AIDS vaccination studies using feline immunodeficiency virus as a model: immunisation with inactivated whole virus suppresses viraemia levels following intravaginal challenge with infected cells but not following intravenous challenge with cell-free virus. Vaccine 1999, 18:119-130.

28. Loewen N, Barraza R, Whitwam T, Saenz DT, Kemler I, Poeschla EM: FIV Vectors. Methods Mol Biol 2003, 229:25I-27I.

29. Stevenson M: HIV nuclear import: What's the flap? Nat Med 2000, 6:626-628.

30. Saenz DT, Poeschla EM: FIV: from lentivirus to lentivector. Gene Med 2004, 6(SuppI I):S95- 104.

31. Mustafa F, Ghazawi A, Jayanth P, Phillip PS, Ali J, Rizvi TA: Sequences intervening between the core packaging determinants are dispensable for maintaining the packaging potential and propagation of feline immunodeficiency virus transfer vector RNAs. IVirol 2005, 79: |38|7-|382|.

32. Segura MM, Kamen A, Garnier A: Downstream processing of oncoretroviral and lentiviral gene therapy vectors. Biotechnol Adv 2006, 24:32I-337.

33. Zhang B, Xia HQ, Cleghorn G, Gobe G, West M, Wei MQ: A highly efficient and consistent method for harvesting large volumes of high-titre lentiviral vectors. Gene Ther 200I, 8: I745-I75I.

34. Landazuri N, Le Doux JM: Complexation with chondroitin sulfate $C$ and polybrene rapidly purifies retrovirus from inhibitors of transduction and substantially enhances gene transfer. Biotechnol Bioeng 2006, 93:146-158.

35. Kang Y, Moressi CJ, Scheetz TE, Xie L, Tran DT, Casavant TL, Ak P Benham CJ, Davidson BL, McCray PB Jr: Integration site choice of a feline immunodeficiency virus vector. J Virol 2006, 80:8820-8823.

36. Monse H, Laufs S, Kuate S, Zeller WJ, Fruehauf S, Uberla K: Viral determinants of integration site preferences of simian immunodeficiency virus-based vectors. I Virol 2006, 80:8145-8I50.

37. Montini E, Cesana D, Schmidt M, Sanvito F, Ponzoni M, Bartholomae C, Sergi Sergi L, Benedicenti F, Ambrosi A, Di Serio C, et al:: Hemat- 
opoietic stem cell gene transfer in a tumor-prone mouse model uncovers low genotoxicity of lentiviral vector integration. Nat Biotechnol 2006, 24:687-696.

38. Sauter SL, Gasmi M: FIV vector systems. Somat Cell Mol Genet 200I, 26:99-129.

39. De Palma M, Montini E, Santoni de Sio FR, Benedicenti F, Gentile A, Medico E, Naldini L: Promoter trapping reveals significant differences in integration site selection between MLV and HIV vectors in primary hematopoietic cells. Blood 2005, I05:2307-2315.

40. Johnston JC, Gasmi M, Lim LE, Elder JH, Yee JK, Jolly DJ, Campbell KP, Davidson BL, Sauter SL: Minimum requirements for efficient transduction of dividing and nondividing cells by feline immunodeficiency virus vectors. J Virol 1999, 73:4991-5000.

4I. D'Souza V, Summers MF: How retroviruses select their genomes. Nat Rev Microbiol 2005, 3:643-655.

42. Moscardini M, Pistello M, Bendinelli M, Ficheux D, Miller JT, Gabus C, Le Grice SF, Surewicz WK, Darlix JL: Functional interactions of nucleocapsid protein of feline immunodeficiency virus and cellular prion protein with the viral RNA. J Mol Biol 2002, 3 I 8:149-159.

43. Zufferey R, Donello JE, Trono D, Hope TJ: Woodchuck hepatitis virus posttranscriptional regulatory element enhances expression of transgenes delivered by retroviral vectors. J Virol 1999, 73:2886-2892.

44. Seganti L, Superti F, Girmenia C, Melucci L, Orsi N: Study of receptors for vesicular stomatitis virus in vertebrate and invertebrate cells. Microbiologica 1986, 9:259-267.

45. DePolo NJ, Reed JD, Sheridan PL, Townsend K, Sauter SL, Jolly DJ, Dubensky TW Jr: VSV-G pseudotyped lentiviral vector particles produced in human cells are inactivated by human serum. Mol Ther 2000, 2:218-222.

46. Verhoeyen E, Cosset FL: Surface-engineering of lentiviral vectors. I Gene Med 2004, 6(Suppl I):S83-94.

47. Sandrin V, Cosset FL: Intracellular versus cell surface assembly of retroviral pseudotypes is determined by the cellular localization of the viral glycoprotein, its capacity to interact with Gag, and the expression of the Nef protein. J Biol Chem 2006, 28 I:528-542.
Publish with Bio Med Central and every scientist can read your work free of charge

"BioMed Central will be the most significant development for disseminating the results of biomedical research in our lifetime. "

Sir Paul Nurse, Cancer Research UK

Your research papers will be:

- available free of charge to the entire biomedical community

- peer reviewed and published immediately upon acceptance

- cited in PubMed and archived on PubMed Central

- yours - you keep the copyright

Submit your manuscript here:

http://www.biomedcentral.com/info/publishing_adv.asp
BioMedcentral 